Supplementary file

\title{
Inhibition of quorum sensing and biofilm formation in Chromobacterium violaceum by fruit extracts of Passiflora edulis
}

\author{
Mahendrarajan Venkatramanan, ${ }^{1, \dagger}$ Pitchaipillai Sankar Ganesh,1, ${ }^{, *}$ \\ Renganathan Senthil,2,3 Jeyachandran Akshay, ${ }^{2}$ Arumugam Veera Ravi,4 \\ Kulanthaivelu Elangaeswaran,5 Jamuna Vadivelu, ${ }^{6}$ Samuthira Nagarajan,7 \\ Kaliyaperumal Rajendran ${ }^{8}$ and Esaki Muthu Shankar ${ }^{1,}$
}

Infection Biology, Department of Life Sciences, School of Life Sciences, Central University of Tamil Nadu, Neelakudi, Thiruvarur 610 o05, India.

${ }^{2}$ Department of Bioinformatics, Marudupandiyar College, Vallam, Thanjavur 613403, India.

3Lysine Biotech Private Limited, Periyar Maniammai University, Periyar Nagar, Vallam, Thanjavur 613403, India.

4Department of Biotechnology, Alagappa University, Karaikudi 630 o03, India.

5Department of Bioinformatics, Alagappa University, Karaikudi 630 003, India.

${ }^{6}$ Department of Medical Microbiology, Faculty of Medicine, University of Malaya, Kuala Lumpur, Malaysia.

7Department of Chemistry, Central University of Tamil Nadu, Neelakudi, Thiruvarur 610 o05, India.

${ }^{8}$ Department of Chemistry, Cape Breton University, Sydney, Nova Scotia, B1P 6L2 Canada. 
Figure S-1: Electrospray ion chromatogram and mass spectrum of EA extract of $P$. edulis bioactive compounds (Peak \# 1 to 20 ) as analysed by GC-MS.

Peak \# 1: 1-Hexadecene

SI:92 Formula:C16 H32 CÁS:629-73-2 MolWeight:224 RetIndex:0

CompName:1-Hexadecene (CAS) Cetene \$ $\$$ 1-Cetene \$ $\$$ n-Hexadec-1-ene \$\$ .alpha.-Hexadecene \$ HEXADECENE-1 \$ Hexadecylene-1 \$\$

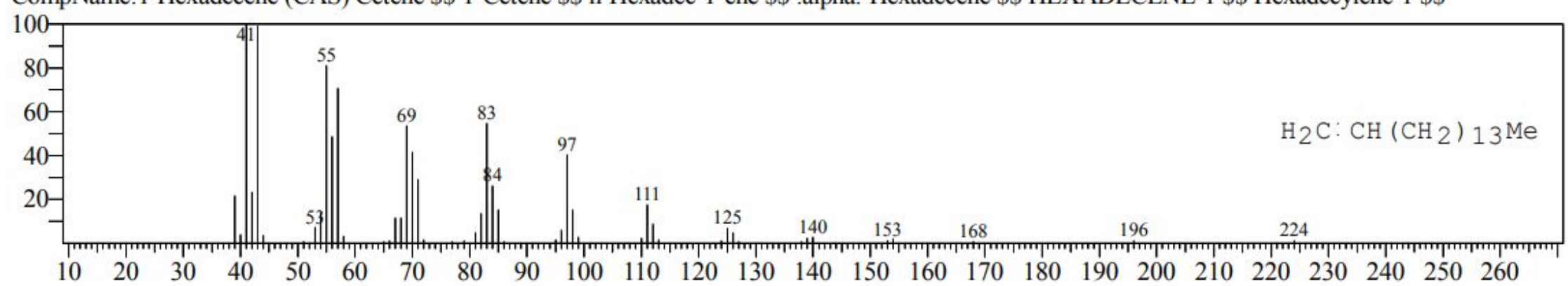

Peak \# 2: 9-octadecene

SI:96 Formula:C18 H36 CÁS:7206-25-9 MolWeight:252 RetIndex:0

CompName:9-Octadecene, (E)-(CAS)

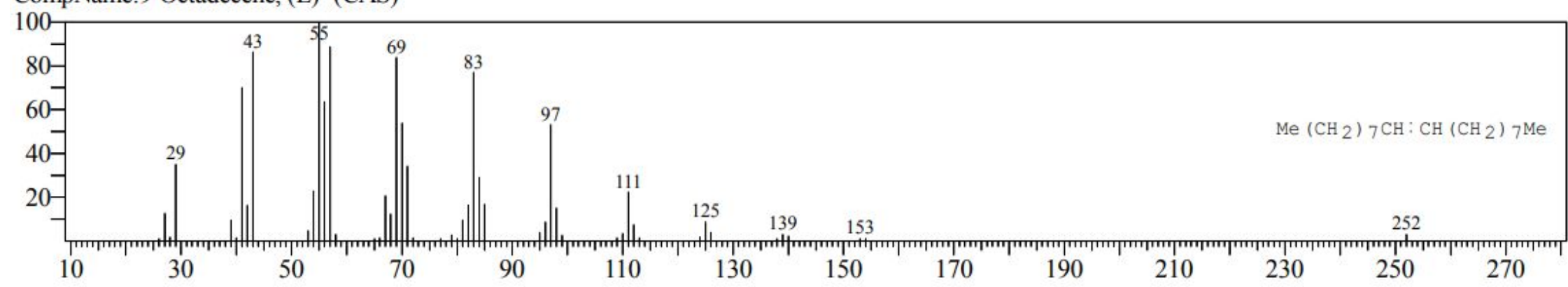


Peak \# 3: Hexadecanoic acid methyl ester

SI:94 Formula:C17 H34 O2 CAS:112-39-0 MolWeight:270 RetIndex:0

CompName:Hexadecanoic acid, methyl ester (CAS) Methyl palmitate \$\$ Methyl hexadecanoate \$\$ Methyl n-hexadecanoate \$\$ Uniphat A60 \$\$ Metholene :

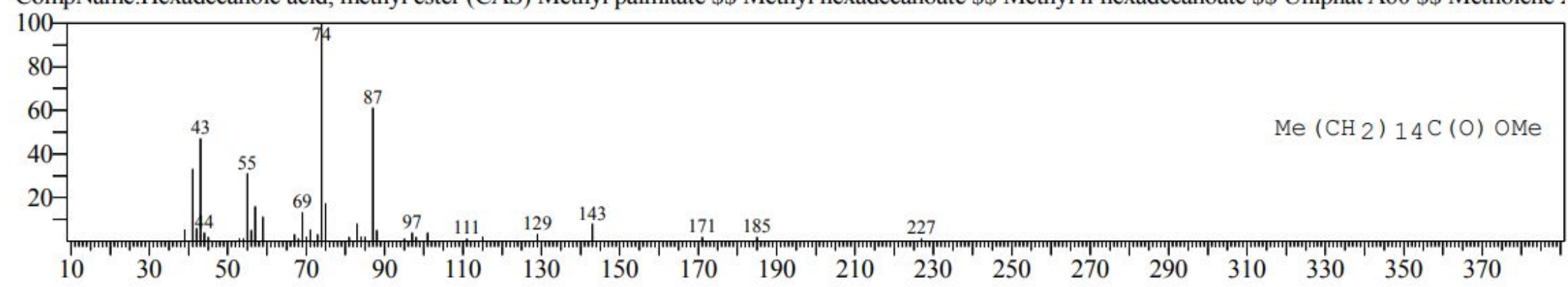

Peak \# 4: 3-tert-butyl-5-hydroxymethyl-cyclohex-2-enyl)-methanol

SI:79 Formula:C12H22O2 CAS:0-00-0 MolWeight:198 RetIndex:0

CompName:(3-TERT-BUTYL-5-HYDROXYMETHYL-CYCLOHEX-2-ENYL)-METHANOL

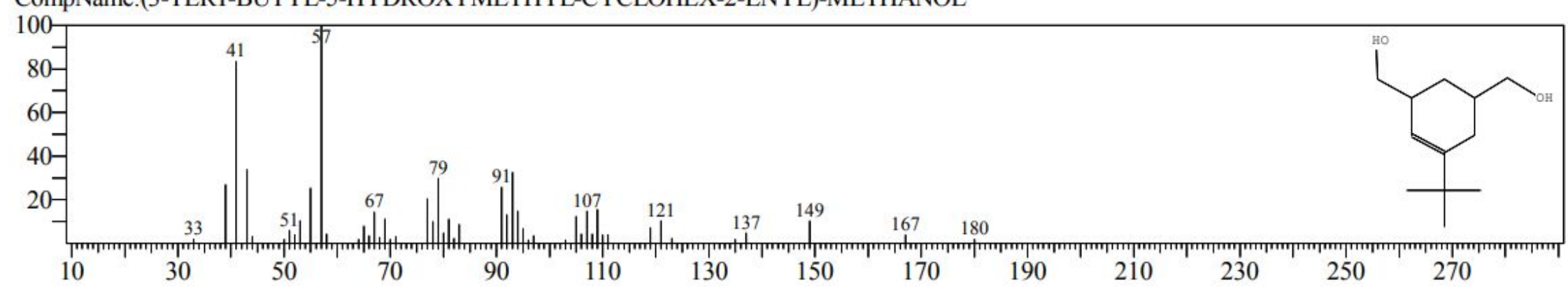


Peak \# 5: Hexadecanoic acid

SI:95 Formula:C16 H32 O2 CAS:57-10-3 MolWeight:256 RetIndex:0

CompName:Hexadecanoic acid (CAS) Palmitic acid \$\$ Palmitinic acid \$ $\$$-Hexadecoic acid \$\$ n-Hexadecanoic acid \$\$ Pentadecanecarboxylic acid \$ $\$ 1-\mathrm{F}$ 100

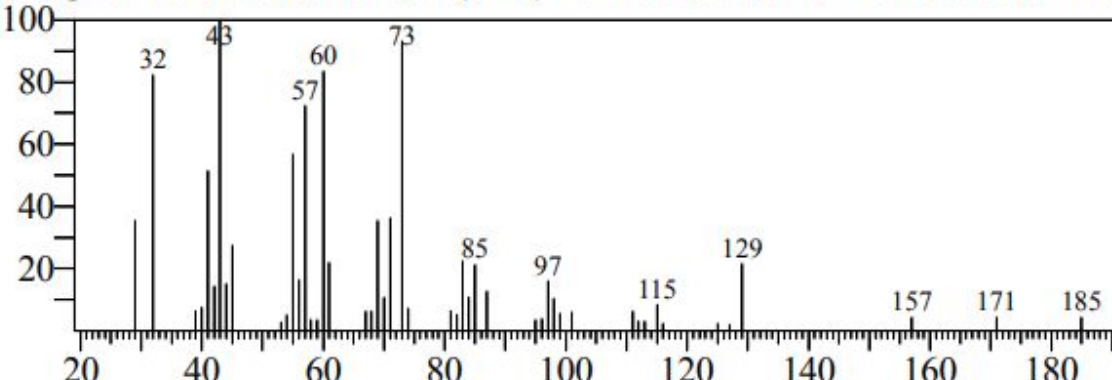

$\mathrm{HO}_{2} \mathrm{C}\left(\mathrm{CH}_{2}\right){ }_{14} \mathrm{Me}$

20

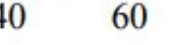

80

$00 \quad 120$

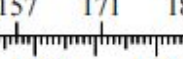
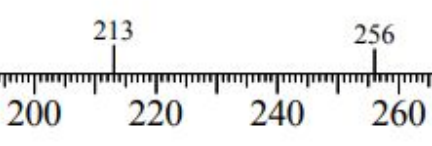

$260 \quad 280$

300

$\mathrm{Me}\left(\mathrm{CH}_{2}\right)_{7} \mathrm{CH}: \mathrm{CH}\left(\mathrm{CH}_{2}\right) 9 \mathrm{Me}$

CÁl:74685-29-3 MolWeight:280 RetIndex:0

CompName:9-Eicosene, (E)- (CAS)

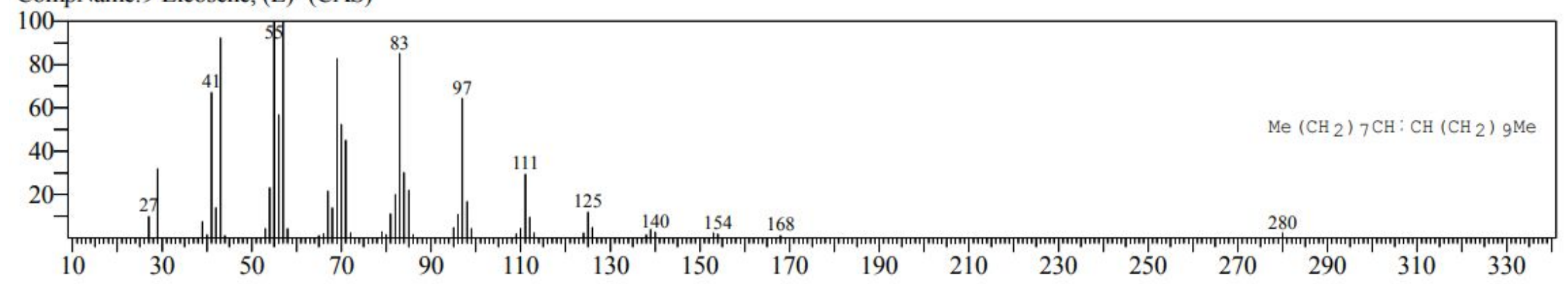


Peak \# 7: n-Hexadecane

SI:86 Formula:C16 H34 CÁS:544-76-3 MolWeight:226 RetIndex:0

CompName:Hexadecane (CAS) n-Hexadecane $\$ \$$ Cetane $\$$ \$ n-Cetane $\$$ Isohexadecane $\$ \$$ HEXADECAN $\$ \$$

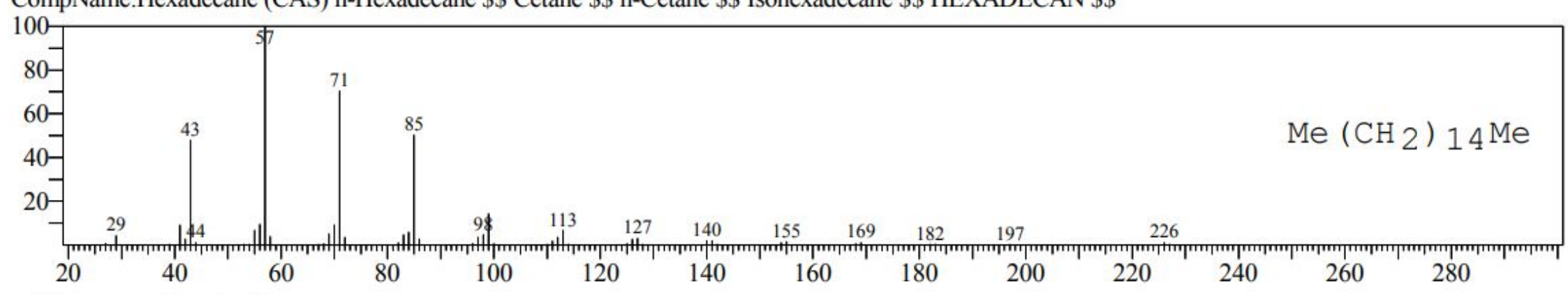

Peak \# 8: 2-Hexadecanol

SI:89 Formula:C16 H34 O CAS:14852-31-4 MolWeight:242 RetIndex:0

CompName:2-Hexadecanol (CAS) Hexadecanol-2 \$\$

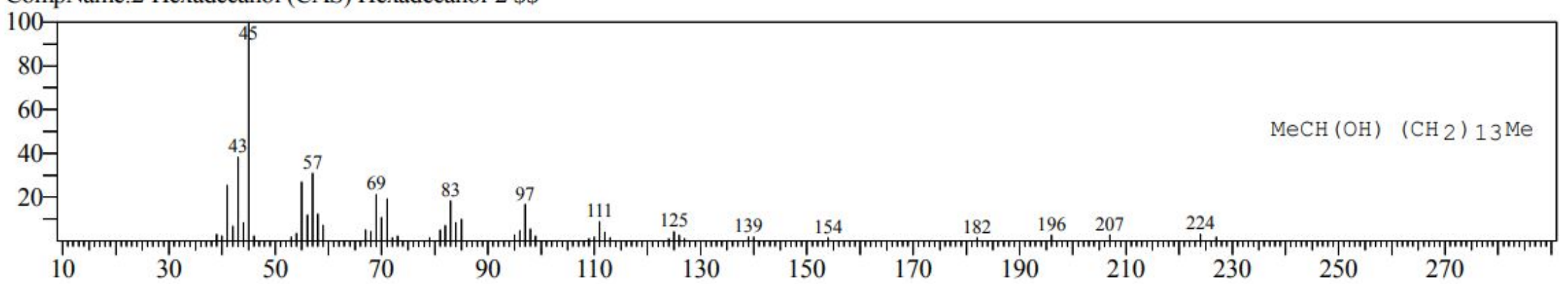

Peak \# 9: 9,12- octadecadienoic acid methyl ester 
SI:93 Formula:C19 H34 O2 CAS:2566-97-4 MolWeight:294 RetIndex:0

CompName:9,12-Octadecadienoic acid, methyl ester, (E,E)- (CAS) Methyl linolelaidate \$ \$ METHYL T9, T12 OCTADECADIENOATE \$ METHYL TRA

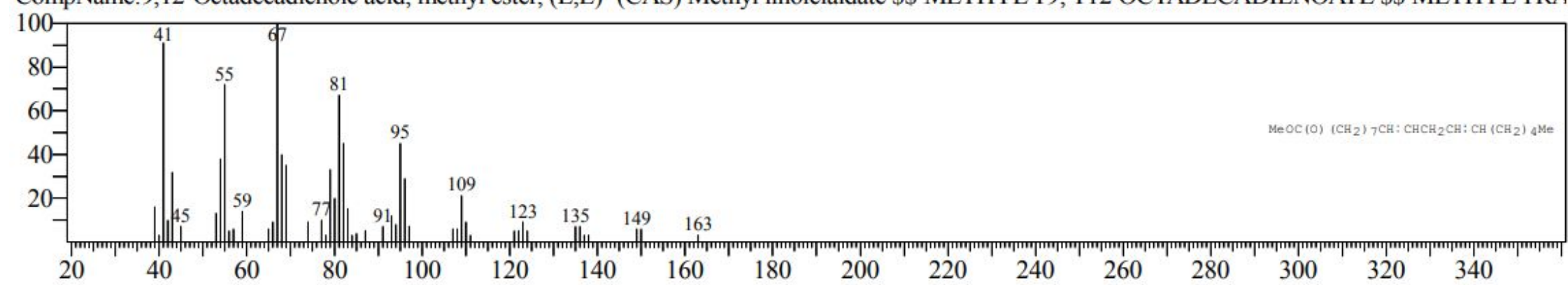

Peak \# 10: 8,11,14- Docosatrienoic acid methyl ester

SI:83 Formula:C23 H40 O2 CAS:56847-02-0 MolWeight:348 RetIndex:0

CompName:8,11,14-Docosatrienoic acid, methyl ester (CAS) METHYL 8,11,14-DOCOSATRIENOATE \$\$

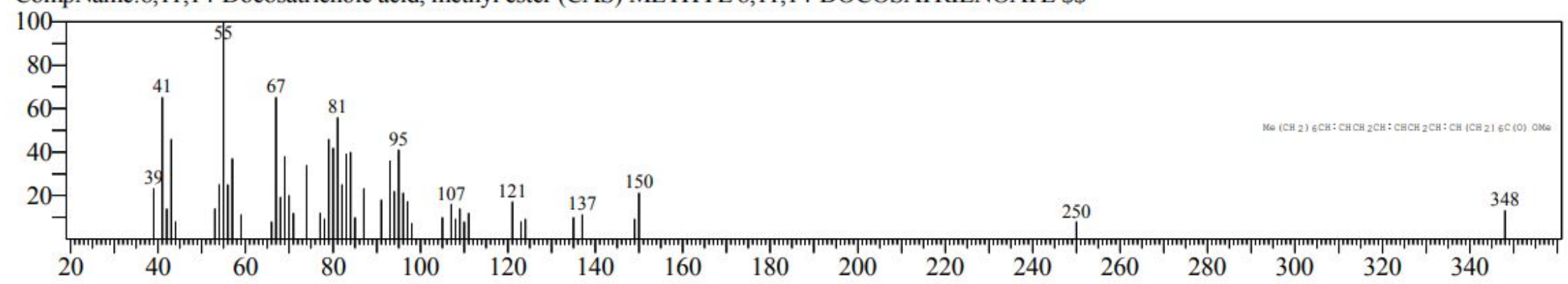


Peak \# 11: 9,12- octadecenoic acid methyl ester

SI:92 Formula:C19 H34 O2 CAS:2566-97-4 MolWeight:294 RetIndex:0

CompName:9,12-Octadecadienoic acid, methyl ester, (E,E)- (CAS) Methyl linolelaidate \$\$ METHYL T9, T12 OCTADECADIENOATE \$ METHYL TRA

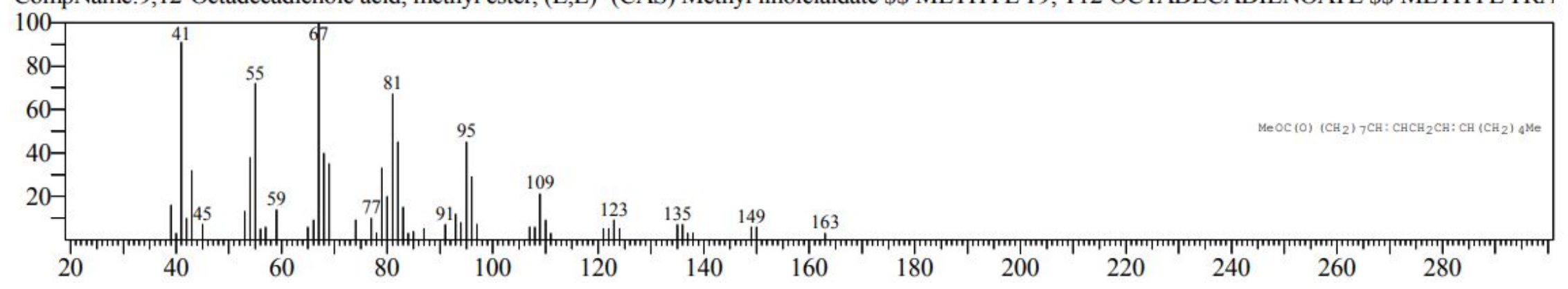

Peak \# 12: 9-octadecenoic acid

SI:93 Formula:C18 H34 O2 CAS:112-80-1 MolWeight:282 RetIndex:0

CompName:9-Octadecenoic acid (Z)- (CAS) Oleic acid \$\$ Red oil \$\$ Oelsauere \$\$ Oleine 7503 \$ Pamolyn 100 \$\$ Emersol 211 \$ Vopcolene $27 \$ \$$ cis-O

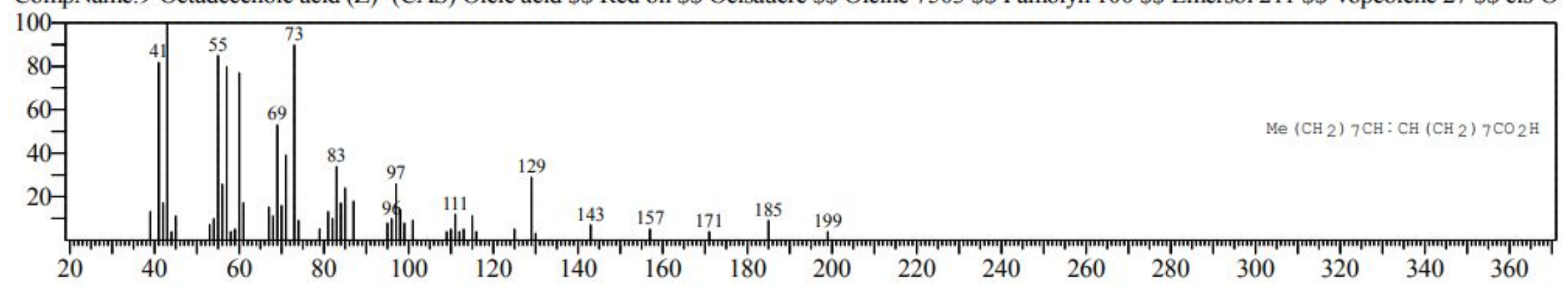


Peak \# 13: Cyclododecane

SI:85 Formula:C12 H24 CAS:294-62-2 MolWeight:168 RetIndex:0

CompName:Cyclododecane (CAS) CYCLODODECAN \$\$

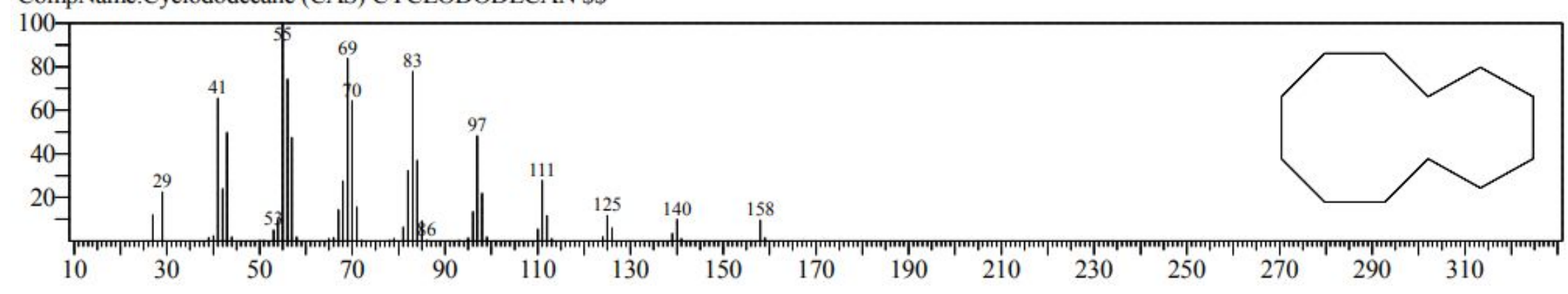

Peak \# 14: Dodecane, 2,7, 10-trimethyl-

SI:89 Formula:C15 H32 CÁS:74645-98-0 MolWeight:212 RetIndex:0

CompName:Dodecane, 2,7,10-trimethyl- (CAS)

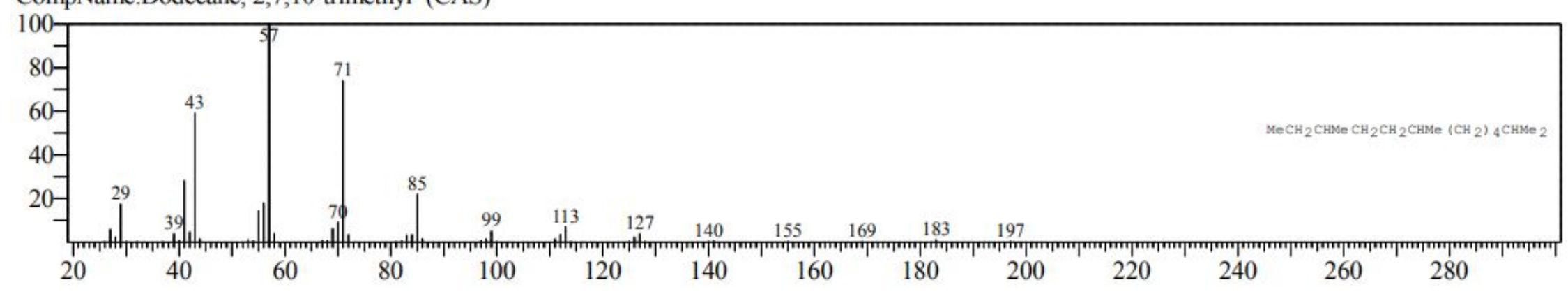


Peak \# 15: n-Octacosane

SI:95 Formula:C28 H58 CÁS:630-02-4 MolWeight:394 RetIndex:0

CompName:Octacosane (CAS) n-Octacosane \$\$

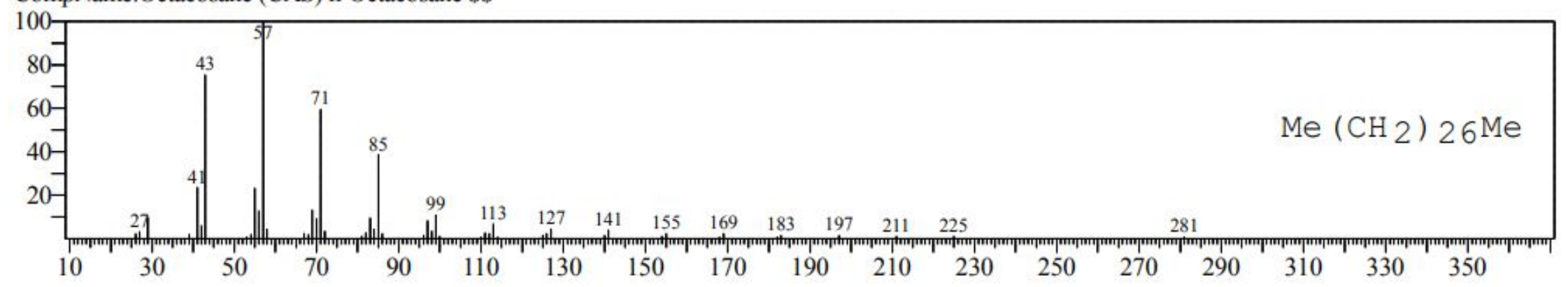

Peak \# 16: n-Tricosane

SI:90 Formula:C23 H48 CÁS:638-67-5 MolWeight:324 RetIndex:0

CompName:Tricosane (CAS) n-Tricosane \$\$

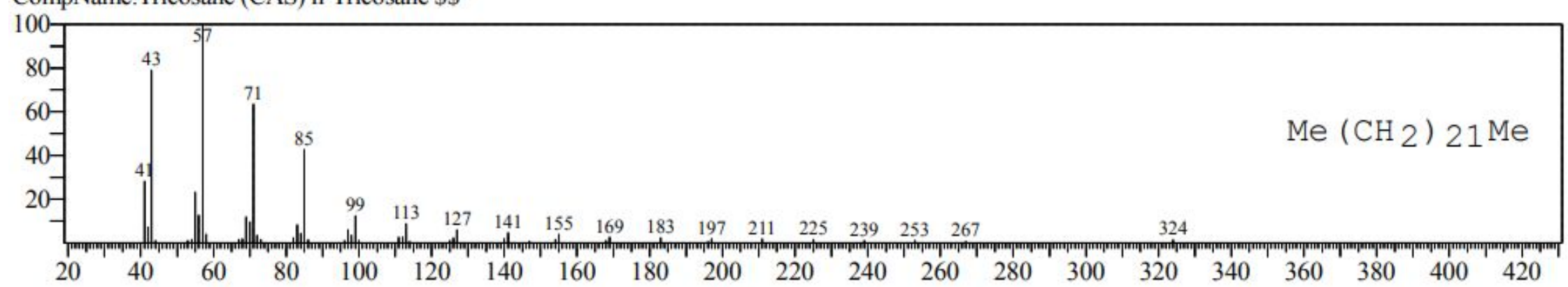


Peak \# 17: 9-Tricosene

SI:88 Formula:C23 H46 CÁS:27519-02-4 MolWeight:322 RetIndex:0

CompName:9-Tricosene, (Z)- (CAS) Muscalure \$\$ cis-9-Tricosene \$\$ (Z)-9-Tricosene \$ $(9 \mathrm{Z})$-Tricosene \$\$

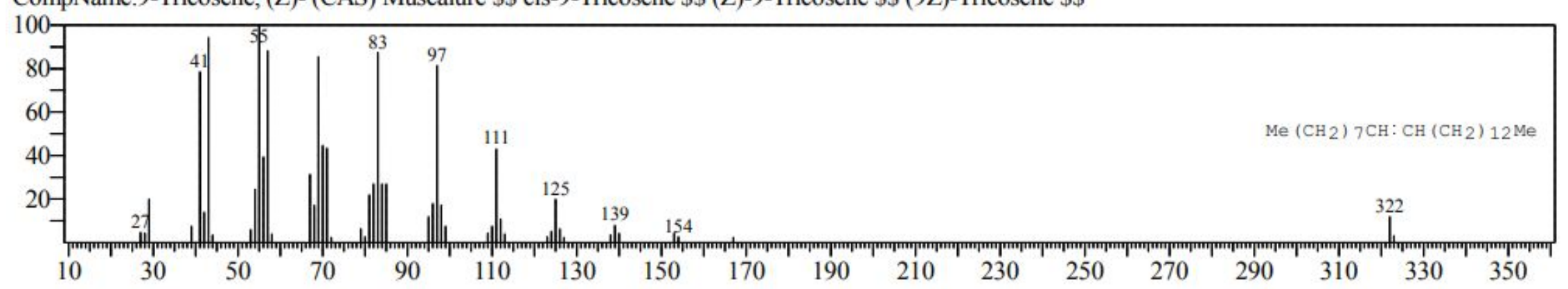

Peak \#18: n- Octacosane

SI:95 Formula:C28 H58 CÁS:630-02-4 MolWeight:394 RetIndex:0

CompName:Octacosane (CAS) n-Octacosane \$\$

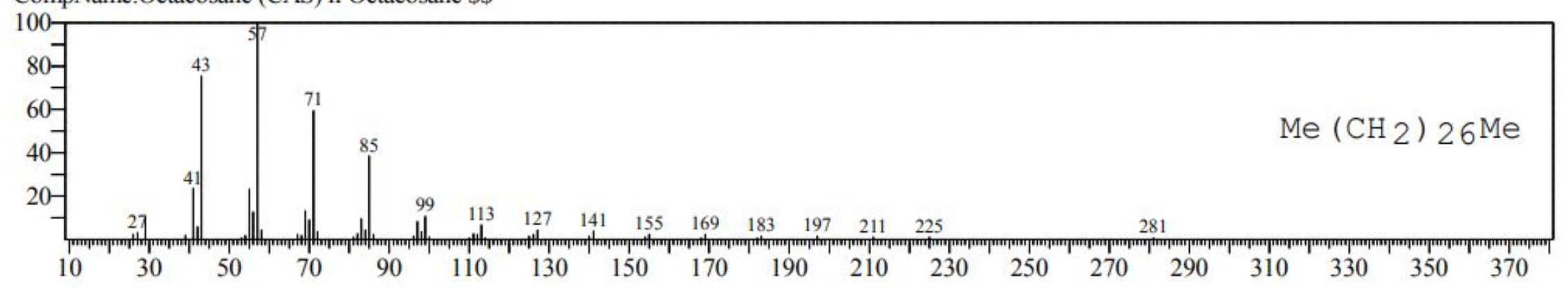


Peak \# 19: Hexadecanoic acid, 2-hydroxy-1-(hydroxymethyl) ethyl ester

SI:89 Formula:C19 H38 O4 CAS:23470-00-0 MolWeight:330 RetIndex:0

CompName:Hexadecanoic acid, 2-hydroxy-1-(hydroxymethyl)ethyl ester (CAS) 2-Monopalmitin \$\$ Palmitin, 2-mono- \$\$ 2-Hexadecanoyl glycerol \$\$ 2-Mc

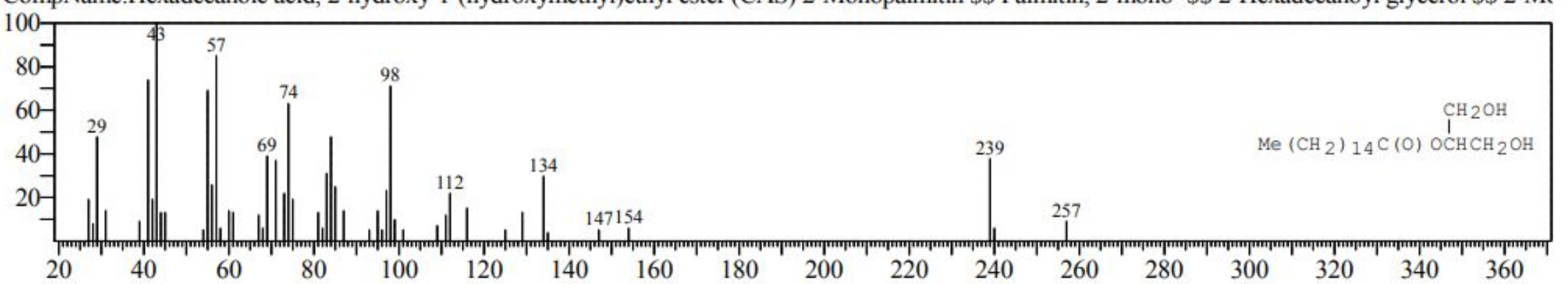

Peak \# 2o: Heptadecane, 2,6,10,15-tetramethyl-

SI:93 Formula:C21 H44 CÁS:54833-48-6 MolWeight:296 RetIndex:0

CompName:Heptadecane, 2,6,10,15-tetramethyl- (CAS) 2,6,10,15-TETRAMETHYLHEPTADECANE \$\$

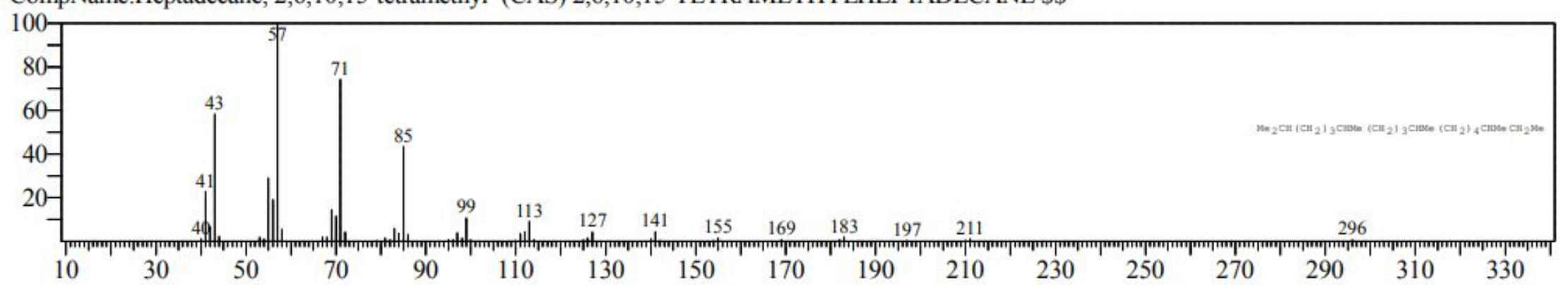

